\title{
Angiotensin Converting Enzyme Inhibition Ameliorates Glomerular Filtration of Macromolecules and Water and Lessens Glomerular Injury in the Rat
}

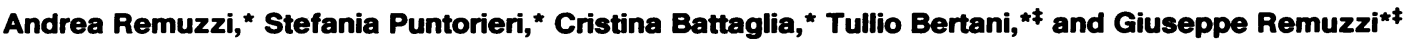 \\ ${ }^{*}$ Mario Negri Institute for Pharmacological Research and ${ }^{\ddagger}$ Division of Nephrology and Dialysis, \\ Ospedali Riuniti di Bergamo, 24100 Bergamo, Italy
}

\section{Abstract}

The effect of enalapril on glomerular hemodynamics and permselectivity and on subsequent sclerosis was studied in male MWF/Ztm rats which spontaneously develop proteinuria and glomerular structural damage. Untreated group 1 and enalapril-treated group 2 (50 $\mathrm{mg} / \mathrm{liter}$, in the drinking water) underwent micropuncture studies after 2 mo of observation. After the same period of treatment, group 3 (untreated) and group 4 (enalapril treated) were used for determination of whole-kidney function and neutral dextran clearances. Group 5 (untreated) and group 6 (enalapril treated) were followed for an additional 4 mo and used for kidney function and morphological studies. Enalapril significantly lowered systolic blood pressure, which was elevated in untreated groups, and significantly reduced proteinuria ( $295 \pm 64 \mathrm{vs} .128 \pm 24 \mathrm{mg} / 24 \mathrm{~h}$ by the end of the study). Despite the reduced renal perfusion pressure, whole-kidney glomerular filtration rate was higher in enalapril-treated than in untreated rats $(0.96 \pm 0.14$ vs. $0.81 \pm 0.10$ $\mathrm{ml} / \mathrm{min}, P<0.05)$ as was the single nephron glomerular filtration rate $(54 \pm 7.1$ vs. $46 \pm 4.0 \mathrm{nl} / \mathrm{min}, P<0.05)$. The single glomerular afferent plasma flow was comparable in both groups. Enalapril reduced mean glomerular capillary hydraulic pressure from the normal value of $51 \pm 1 \mathrm{mmHg}$ (untreated rats) to a value lower than normal $(44 \pm 1 \mathrm{mmHg}, P<0.001)$ These hemodynamic changes were associated with a significant reduction in afferent $(\approx 23 \%)$ and efferent $(\approx 26 \%)$ arteriolar resistance. The mean ultrafiltration coefficient was two times higher in the enalapril $(0.126 \pm 0.027 \mathrm{nl} / \mathrm{s}$ per $\mathrm{mmHg})$ than in the untreated group $(0.061 \pm 0.023 \mathrm{nl} / \mathrm{s}$ per $\mathrm{mmHg})$. The clearance of dextran macromolecules relative to that of inulin was significantly reduced for all molecular sizes studied (26-64 $\AA$ ) in enalapril-treated vs. untreated rats. Theoretical analysis of dextran fractional clearances using a heteroporous model of neutral solute transport across the glomerular capillary wall indicated that enalapril affected glomerular membrane size selective properties, reducing uniformly the radius of hypothetical membrane pores. Enalapril treatment also significantly limited $(P<0.01)$ the development of glomerular structural lesions (mean percentage of sclerotic glomeruli was $4.2 \pm 3.5 \%$ [treated] vs. $28 \pm 15 \%$ [untreated] rats at the end of the study) as well as tubulo-interstitial damage. These results suggest that the protective effect of enalapril on the develop-

Address reprint requests to Dr. A. Remuzzi, Istituto di Ricerche Farmacologiche Mario Negri, Via Gavazzeni, 11, 24100 Bergamo, Italy.

Received for publication 3 August 1989 and in revised form 2 October 1989.

J. Clin. Invest.

(c) The American Society for Clinical Investigation, Inc.

0021-9738/90/02/0541/09 \$2.00

Volume 85, February 1990, 541-549 ment of proteinuria and glomerular sclerosis in this model is due to its property of ameliorating size selectivity and hydraulic permeability of the glomerular capillaries. (J. Clin. Invest. 1990. 85:541-549.) angiotensin converting enzyme • glomerular hemodynamics • glomerulosclerosis • proteinuria

\section{Introduction}

Elevation in glomerular capillary hydraulic pressure and plasma flow rate is associated with progressive proteinuria and eventual glomerulosclerosis in several rat models of kidney damage including extensive ablation of the renal mass and streptozotocin-induced diabetes (1-4). In both models the normalization of glomerular capillary hydraulic pressure by the angiotensin I converting enzyme (CE) ${ }^{1}$ inhibitor enalapril protected against the subsequent development of glomerular structural injury and proteinuria $(5,6)$. These observations prompted the suggestion that glomerular capillary hypertension is crucial to the development of glomerulosclerosis and renal function deterioration in these models of renal disease (7). That an angiotensin CE inhibitor may protect against progressive glomerulosclerosis independently of its property of lowering glomerular hypertension has been recently suggested by Fogo et al. (8) in two established models of glomerulosclerosis induced in rats by puromycin or adriamycin injection. In both models chronic administration of captopril protected against the development of glomerulosclerosis; however, intraglomerular capillary pressure, measured in early stages of the glomerulopathy, was not modified by the angiotensin CE inhibitor. On the same line of investigation, Morelli et al. (9) have recently documented that, in humans with insulin-dependent diabetes, enalapril exerts an antiproteinuric effect by shifting glomerular pores towards a smaller size, thus enhancing the size-selective barrier.

We have previously investigated single-glomerular hemodynamics in a strain originally selected from Munich-Wistar rats by Frömter (MWF/Ztm) for its high number of superficial glomeruli (10). Males of this strain develop spontaneous proteinuria (11) and glomerulosclerosis with age (Remuzzi, A., unpublished observation), whereas females are protected from glomerular damage. Unlike in animals with surgical reduction of functioning nephrons or given renal toxins, in this model glomerular damage develops spontaneously and thus represents an ideal condition to study the mechanism(s) underling renal disease progression.

The present study was designed with the following aims: $(a)$ to establish whether enalapril protects against the development

1. Abbreviations used in this paper: $\mathrm{AP}$, mean arterial pressure; $\mathrm{CE}$, converting enzyme; $\mathrm{FF}$, filtration fraction; $\mathrm{GV}$, glomerular volume; MWF/Ztm, Munich-Wistar-Frömter (rats); PAH, p-aminohippuric acid; RPF, renal plasma flow; SBP, systolic blood pressure; SN, single nephron. 
of glomerular injury in a rat model of renal disease characterized by normal glomerular capillary pressure (11-13); $(b)$ to elucidate the pathophysiological basis of such phenomenon by the study of glomerular hemodynamics and size-selective function.

\section{Methods}

47 male MWF/Ztm rats of $10 \mathrm{wk}$ of age were used in these studies. Rats, originally supplied by $H$. Hackbarth $(10,11)$, were bred and raised in our facilities. All animals were allowed free access to food (standard rat laboratory diet containing $20 \%$ protein by weight) and water. Groups 1, 3, and 5 received no specific therapy and are later referred to as control groups. Groups 2, 4, and 6 were treated with the angiotensin I CE inhibitor enalapril (Merck Sharp \& Dohme Italia $\mathrm{SpA}$, Rome, Italy), at a dose of $50 \mathrm{mg} /$ liter in drinking water throughout the duration of the study.

Untreated group $1(n=8)$ and enalapril-treated group $2(n=10)$ underwent micropuncture study and morphological evaluation after 2 mo of observation. After the same time of treatment, group 3 (untreated, $n=7$ ) and group 4 (enalapril treated, $n=8$ ) were used for determination of whole-kidney function and neutral dextran clearances. Group 5 (untreated, $n=8$ ) and group 6 (enalapril treated, $n=6$ ) were followed for an additional 4 mo at which time whole-kidney function was measured and renal tissue was processed for morphological studies and glomerular volume (GV) estimation. During the observation period systolic blood pressure (SBP) was measured every 2 wk in all rats using the tail cuff method (14). The urinary protein excretion rate was measured at the same time interval. Protein concentrations in urine were determined by the Coomassie blue $\mathrm{G}$ dye-binding method (15).

Micropuncture studies. Micropuncture studies were performed with the method previously described (11). Briefly, rats were anesthetized with Inactin ( $100 \mathrm{mg} / \mathrm{kg}$ body wt, i.p.), placed on a constant-temperature micropuncture table and tracheostomized. The left femoral artery was catheterized for blood sampling and for continuous arterial pressure (AP) measurement, using a pressure transducer and a writing recorder (Battaglia Rangoni, Bologna, Italy). Polyethylene catheters were inserted into the left jugular and left femoral vein for infusion of inulin solution ( $7 \%$, dissolved in $0.9 \% \mathrm{NaCl}$ ) and isoncotic rat plasma. The left kidney was then exposed through a subcostal incision, a polyethylene catheter was inserted into the left ureter, and the kidney was placed in a Lucite holder and bathed with isotonic $\mathrm{NaCl}$. Isoncotic rat plasma, obtained from a littermate immediately before the operation, was infused during the experiment to compensate surgically induced loss of plasma volume as previously described $(11,12)$.

After a 1-h equilibration period exactly timed tubular fluid samples were collected from proximal convolutions of five to six separate nephrons using sharpened glass pipettes. Three to five samples of efferent arteriolar blood were withdrawn from superficial star vessels using sharpened oil-filled pipettes. Time-averaged hydraulic pressure was measured in surface glomerular capillaries, proximal tubules, and in first-order peritubular capillaries using sharpened glass pipettes and a servo-nulling micropipette pressure system (model 5, Instrumentation for Physiology and Medicine, San Diego, CA). During micropuncture measurements three timed urine collections, of 20-30 min each, were started. Blood samples were obtained at the midpoint of each clearance period for determination of hematocrit and plasma concentrations of inulin and total proteins.

The volume of tubular fluid samples was measured from the length of the fluid column in a constant bore capillary tube of known diameter. The inulin concentration in tubular fluid was determined, usually in duplicate, by the method of Vurek and Pegram (16). Protein concentrations in efferent arteriolar and femoral arterial plasma samples were determined, usually in duplicate, using an orthophthalaldehyde technique (17) adapted for small sample volumes as described by Viets et al. (18). Inulin concentrations in plasma and urine samples were measured using the anthrone method of Fuehr et al. (19). Total protein concentration in arterial plasma was determined by the method of Lowry et al. (20). Glomerular filtration rate (GFR), measured as inulin clearance, was calculated using the standard formula. Calculations of single-nephron (SN)GFR, colloid osmotic pressure in afferent and efferent arterioles $\left(\Pi_{A}, \Pi_{E}\right), S N$ filtration fraction (FF), single glomerular afferent plasma flow $\left(Q_{A}\right)$, and afferent and efferent arteriolar resistance $\left(\mathbf{R}_{A}, R_{E}\right)$ were performed using equations described in detail previously (21). Calculation of the ultrafiltration coefficient $\left(K_{f}\right)$ was performed according to the model of glomerular ultrafiltration of Deen et al. (22).

Whole-kidney function and dextran clearance studies. Inulin, paminohippuric acid (PAH), and neutral dextran clearance experiments were performed in rats of group 3 and 4 as previously described (23). Rats were anesthetized as described for micropuncture study and placed on a constant-temperature table. A solution of $0.9 \% \mathrm{NaCl}$ containing $5 \%$ inulin, $0.2 \% \mathrm{PAH}$, and ${ }^{125} \mathrm{I}$-dextrans $(\approx 15 \mu \mathrm{Ci}$ per animal) was infused through the femoral vein at a constant rate of $0.045 \mathrm{ml} /$ min. AP in left femoral artery was continuously monitored during the clearance experiment and urine was collected by bladder incannulation. 40-50 min after the beginning of surgical preparation, three timed clearance periods of about $30 \mathrm{~min}$ each were started. Blood samples were drawn at the midpoint of each clearance period. Inulin and total protein concentrations in plasma and urine samples were determined as described for the micropuncture study. PAH concentrations in plasma and urine samples were determined by the method of Smith et al. (24). GFR and renal plasma flow (RPF) were calculated as inulin and PAH clearance, respectively. Animals of groups 5 and 6 underwent inulin and PAH clearance studies, after 6 mo of observation, for whole-kidney function evaluation with the methodological procedure above described for dextran clearance studies but without dextran infusion.

Preparation and separation of radiolabeled dextrans. Radiolabeled neutral dextran of a wide molecular size distribution were prepared according to the method of Yoshioka et al. (25). Briefly, a mixture of three dextran fractions of known molecular weight (dextrans T10, T40, and T70, Pharmacia, Uppsala, Sweden) were oxidized using $\mathrm{NaIO}_{4}$ and incubated with tyramine. Purified tyraminil-dextran was radiolabeled with ${ }^{125}$ I by incubation with Iodo-Beads (Pierce Chemical Co., Rockford, IL). Unincorporated ${ }^{125}$ I was removed by gel chromatography (Sephadex G25 column, PD-10, Pharmacia). The ${ }^{125}$ I-dextran solution was then filtrated and stored at $4^{\circ} \mathrm{C}$ before use.

The fractionation of ${ }^{125}$ I-neutral dextran in plasma and urine samples, collected during the first clearance period, into graded molecular size was accomplished by gel permeation chromatography using a Ultrogel AcA 34 (LKB Produkter, Bromma, Sweden) column (60 $\times 1.6 \mathrm{~cm})$, as described previously $(23,25)$. Column calibration was performed periodically using nonradioactive dextran fractions of known molecular weight (dextrans T10, T40, T70, Pharmacia). During sample separation, fractions of $\sim 2 \mathrm{ml}$ were automatically collected and the radioactivity of each fraction was measured by a $\gamma$ counter (Beckman Instruments, Inc., Palo Alto, CA). Effective molecular radii of neutral dextran molecules contained in individual fractions were determined as described by Granath and Kvist (26). Fractional clearance of neutral dextrans (effective molecular radius ranging from 26 to $64 \AA$ ) was calculated as: $\theta_{\mathrm{D}}=(U / P)_{\mathrm{D}} /(U / P)_{\mathrm{IN}}$, where $(U / P)_{\mathrm{D}}$ and $(U / P)_{\mathrm{IN}}$ are the urine-to-plasma concentration ratios of neutral dextrans and inulin, respectively.

Theoretical analysis of membrane pore structure. Membrane poresize parameters were calculated using the model of Deen et al. (27) as described in a previous study (23). This model, derived for an arbitrary distribution of membrane pore-sizes, was previously used to shown that representation of the glomerular membrane as perforated by a lognormal distribution of pore radii gives the most accurate result in simulating dextran clearance measurements in the rat (23). The assumed lognormal distribution of pore radii was defined as follows:

$g(r)=\frac{1}{\sqrt{2 \pi} r \ln s} \exp \left[-\frac{1}{2}\left(\frac{\ln r-\ln u}{\ln s}\right)^{2}\right]$ 
where $u$ and $s$ are the mean and the standard deviation of the corresponding normal probability distribution. We have then defined another pore-size parameter, $r^{*}(1 \%)$, to quantify the presence of largest membrane pores, through which large macromolecules and proteins are filtered. As described previously (23), $r^{*}(1 \%)$ is the pore radius for which a fraction of $1 \%$ of filtrate volume passes through pores with radii ranging from $r^{*}$ and $\infty$.

For specified values of intrinsic membrane parameters $(u$ and $s)$ and the four determinants of glomerular ultrafiltration $\left(\mathrm{Q}_{\mathrm{A}}, \mathrm{C}_{\mathrm{A}}, \overline{\Delta \mathrm{P}}\right.$, and $K_{f}$ ) the adopted theoretical model allows the computation of $\theta_{D}$ for a solute of given molecular radius $r$. The values of $Q_{A}$ and $C_{A}$, adopted for the calculations, were those experimentally determined during the dextran clearance studies, respectively, as PAH clearance and total protein concentration in arterial plasma. The values of $\overline{\Delta P}$, not measured during dextran clearance studies, were assumed to be equal to the mean values observed during micropuncture studies in superficial glomeruli of animals of groups 1 and 2, that were comparable for age and treatment to animals of groups 3 and 4 . An estimation of $K_{\mathrm{f}}$ for both kidneys was calculated using the model of glomerular ultrafiltration described previously (22) and the experimental values of GFR, $Q_{A}, C_{A}$ together with the assumed values of $\overline{\Delta \mathrm{P}}$. Optimal parameters values for $u$ and $s$ were calculated for each animal that underwent dextran clearance study using a computer program for nonlinear regression fitting (28).

Morphological studies. After micropuncture (groups 1 and 2) or whole-kidney function studies (groups 5 and 6), fragments of the left kidney were fixed in Dubosq-Brazil fluid, as described previously (29), and embedded in paraffin. Sections $3 \mu \mathrm{m}$ thick were stained with Masson's trichrome, with hematoxylin and eosin, and by the periodicacid Schiff techniques. Sections including superficial and juxtamedullary glomeruli were evaluated. At least 100 glomeruli were examined for each animal and the percentage of glomeruli affected by focal or global glomerular sclerosis was determined. Tubular changes (atrophy, casts, and dilatation) and interstitial fibrosis and inflammation were graded from 0 to $4+(0=$ no changes; $1+=$ changes affecting $<25 \%$ of sample; $2+=$ changes affecting $25-50 \%$ of sample; $3+=$ changes affecting $50-75 \%$ of sample; $4+=$ changes affecting $>75 \%$ of sample). Renal biopsies were analyzed by the same pathologist blind to the nature of the experimental groups.

GV determination was performed as described by Weibel (30) with slight modifications. Histological sections were projected on a drawing table (enlargement $\times 325$ ), using a light microscope and a drawing tube. The outline of the capillary tuft was manually traced and its area measured using an image analysis system based on a digital scanner (model 300FB, Abaton, Fremont, CA) connected to a personal computer (Macintosh II, Apple Computer, Inc., Cupertino, CA). Exact enlargement was calculated from the direct measure of a reference grid with a micrometer eyepiece (Nachet, Levallois-Perret, France). Mean glomerular random cross-sectional area $\left(A_{\mathrm{m}}\right)$ was determined in at least 50 systematically sampled glomeruli per animal. Average $\mathbf{G V}$ of individual animals was calculated using the equation derived by Weibel (30): $\mathrm{GV}=(\beta / k)\left(A_{\mathrm{m}}\right)^{3 / 2}$, where $k=1.1$ is a size distribution coefficient and $\beta=1.38$ is the shape coefficient for spheres (the assumed shape of glomeruli).

Statistical analysis. All results are expressed as mean \pm 1 SD. Data were analyzed using Student's $t$ test for unpaired data, two-way analysis of variance or two-factor mixed design as appropriate. Significance level of differences between individual group means, subjected to the analysis of variance, was established using Tukey-Cicchetti test for multiple comparisons (31). Estimates of renal damage by morphological studies were compared with Mann-Whitney test for non parametric data. Statistical significance level was defined as $P<0.05$.

\section{Results}

Systemic blood pressure. As shown in Fig. 1, untreated male MWF/Ztm rats (group 5) showed systemic hypertension at the

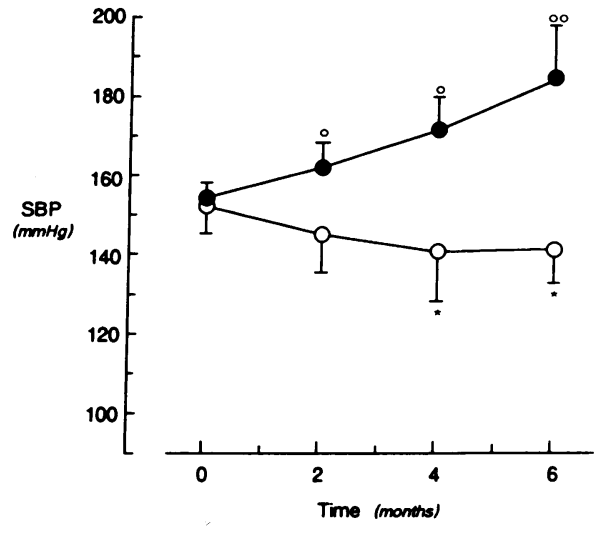

Figure 1. SBP measured in conscious rats during 6 mo of observation in (๑ control group 5 and (o) enalapril-treated group 6 . In untreated rats SBP significantly increased during the observation, whereas in enalapril-treated rats SBP tended to decrease. Values are means \pm SD. (o) $P<0.05$ vs. same group at months 0 . (০) $P<0.01$ vs. same group at months $0 .(*) P<0.01$ vs. control group at the same time point.

beginning of the experimental period $(154 \pm 4 \mathrm{mmHg})$. Blood pressure (SBP) further increased with time reaching the value of $184 \pm 17 \mathrm{mmHg}$ by the end of the study. In rats treated with the CE inhibitor enalapril (group 6) SBP tended to decrease, during the observation period, although the reduction was not statistically significant $(151 \pm 6$ vs. $141 \pm 7 \mathrm{mmHg})$.

Proteinuria. Urinary protein excretion rates for untreated (group 5) and enalapril-treated rats (group 6) during the observation period are reported in Fig. 2. Untreated rats developed massive proteinuria with the age reaching the value of $295 \pm 64$ $\mathrm{mg} / 24 \mathrm{~h}$ by the end of the study. Enalapril treatment significantly reduced the urinary protein excretion rate, as compared with that of untreated rats at the same time point, from the 2nd to the 6th mo of observation; proteinuria was completely prevented in enalapril-treated group 6 during the first 2 mo of treatment, then showed a tendency to increase at the 4th mo,

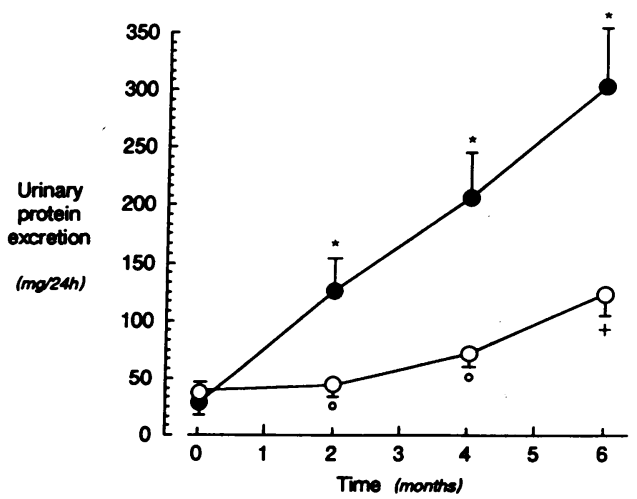

Figure 2. Urinary protein excretion rate during $6 \mathrm{mo}$ of observation in (๑) control (group 5) and (0) enalapril-treated (group 6) rats. Untreated rats developed massive proteinuria with age, whereas in enalapril-treated rats urinary protein excretion was significantly prevented. Values are means \pm SD. $(*) P<0.01$ vs. same group at month 0 . (0) $P<0.01$ vs. control group at the same time point. $(+) P$ $<0.01$ vs. control group at the same time point and vs. same group at month 0 . 
Table I. Whole-Kidney and Single-Nephron Functional Parameters

\begin{tabular}{lccccccccc}
\hline \multicolumn{1}{c}{ Group } & Body wt & Kidney wt & Hct & $\overline{\mathrm{AP}}$ & $\mathrm{GFR}$ & $\overline{\mathrm{P}}_{\mathrm{GC}}$ & $\mathrm{P}_{\mathrm{T}}$ & $\overline{\Delta \mathrm{P}}$ & $\mathrm{P}_{\mathrm{E}}$ \\
\hline & $g$ & $g$ & $\%$ & $m m H g$ & $m l / m i n$ & $m m H g$ & $m m H g$ & $m m H g$ & $m m H g$ \\
1. Control $(n=8)$ & $378 \pm 30$ & $1.10 \pm 0.07$ & $50 \pm 1$ & $129 \pm 9$ & $0.81 \pm 0.10$ & $51 \pm 1$ & $13 \pm 1$ & $38 \pm 2$ & $21 \pm 2$ \\
2. Enalapril $(n=10)$ & $376 \pm 18$ & $1.11 \pm 0.08$ & $51 \pm 1$ & $110 \pm 6$ & $0.96 \pm 0.14$ & $44 \pm 1$ & $13 \pm 1$ & $31 \pm 2$ & $20 \pm 1$ \\
$P$ 1 vs. 2 & $\mathrm{NS}$ & $\mathrm{NS}$ & $\mathrm{NS}$ & $<0.001$ & $<0.05$ & $<0.001$ & NS & $<0.001$ & $\mathrm{NS}$ \\
& & & & & & & &
\end{tabular}

Abbreviations used in this table: $\pi_{A}, \pi_{E}$ afferent and efferent colloid osmotic pressure; $C_{A}, C_{E}$, afferent and efferent arteriolar plasma protein concentration; Hct, hematocrit; $K_{\mathrm{f}}$, ultrafiltration coefficient; $\mathrm{P}_{\mathrm{E}}$, efferent arteriolar hydraulic pressure; $\mathrm{P}_{\mathrm{T}}$, proximal tubule hydraulic pressure; $Q_{A}$, single glomerular afferent plasma flow; $R_{A}, R_{E}$, afferent and efferent arteriolar resistance; $R_{T}$, total arteriolar resistance $\left(R_{A}+R_{E}\right)$. Values are mean $\pm \mathrm{SD}$

and reached the mean value of $128 \pm 24 \mathrm{mg} / 24 \mathrm{~h}$ by the end of the study. Urinary protein excretion in animals that underwent dextran clearance studies (groups 3 and 4 ) was comparable to that observed in groups 5 and 6 at month 2 (see Table II and Fig. 2).

Micropuncture studies. Mean values of whole-kidney and single-nephron functional parameters measured in groups 1 and 2, at the 2nd mo of observation, are summarized in Table I and in Fig. 3. No differences were observed for body weight, kidney weight, and hematocrit between the two animal groups. $\overline{\mathrm{AP}}$ under anesthesia was significantly reduced $(P<0.001)$ in enalapril-treated (group 2) as compared with untreated rats (group 1) paralleling SBP measurements in awake animals (see Fig. 1). Despite the lower renal perfusion pressure, total kidney GFR was higher in treated than in untreated rats $(0.96 \pm 0.14$

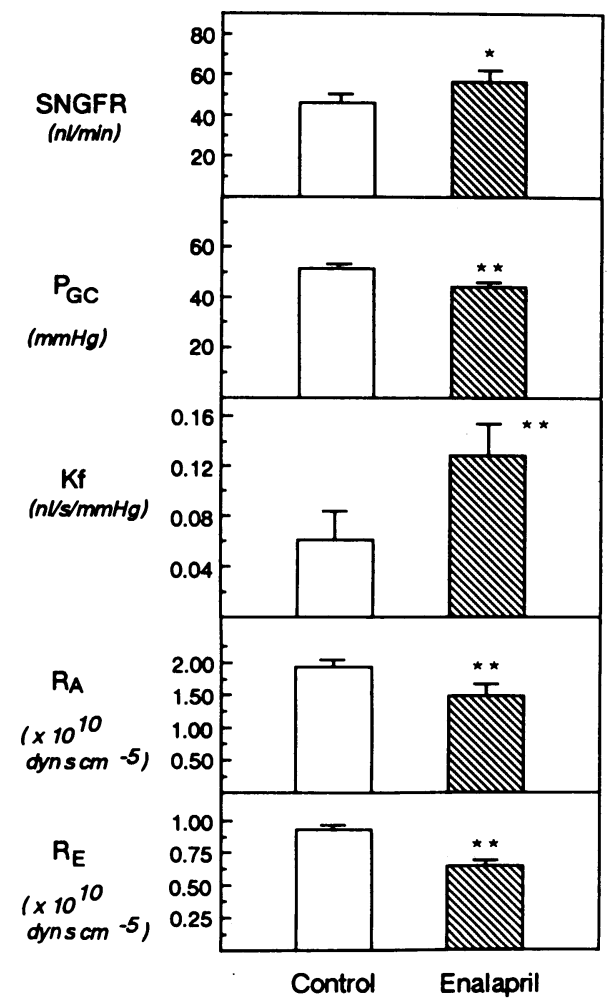

Figure 3. Single glomerular functional parameters in control and in enalapril-treated rats after 2 mo of observation. Bars represent means \pm SD. (*) $P<0.05$ vs. control group. (**) $P<0.001$ vs. control group. vs. $0.81 \pm 0.10 \mathrm{ml} / \mathrm{min}$, respectively, $P<0.05$ ), although when whole-kidney GFR was factored by kidney weight the difference did not reach statistical significance. As reported in a previous study, untreated rats (group 1) exhibited a normal value of mean glomerular capillary hydraulic pressure $\left(P_{G C}\right.$ $=51 \pm 1 \mathrm{mmHg}$ ), comparable to values reported in literature for Munich-Wistar rats in other laboratories. In enalapriltreated rats, $P_{\mathrm{GC}}$ was significantly reduced $(44 \pm 1 \mathrm{mmHg}, P$ $<0.001)$ as compared with untreated animals. Since proximal tubule hydraulic pressure was comparable in the two animal groups, the calculated mean transmembrane hydraulic pressure difference $(\overline{\Delta \mathrm{P}})$ was significantly lower $(P<0.001)$ in rats treated with $C E$ inhibitor than in untreated animals $(31 \pm 2$ vs. $38 \pm 2 \mathrm{mmHg}$, respectively). Values for hydraulic pressure in first-order peritubular capillaries as well as afferent and efferent arteriolar plasma protein concentration and colloid osmotic pressure were not different in the two groups.

Despite the lower mean value of $\overline{\Delta \mathbf{P}}$, enalapril-treated rats exhibited higher values of mean SNGFR (on average $54 \pm 7.1$ $\mathrm{nl} / \mathrm{min})$ in comparison with the untreated group $(46 \pm 4.0 \mathrm{nl} /$ min, $P<0.05)$ paralleling the corresponding mean values of whole-kidney GFR. Mean SNFF and $Q_{A}$ were numerically higher in the enalapril-treated group although the difference did not reach statistical significance. As reported in previous studies on other models of progressive glomerular disease $(5$, 32) CE inhibition significantly affected glomerular arteriolar tone by significant reduction of both afferent $(\approx 23 \%)$ and efferent $(\approx 26 \%)$ arteriolar resistance. As a result total arteriolar resistance was reduced $(P<0.001)$ of $\sim 24 \%$ in enalapriltreated rats as compared with untreated animals. Calculation of unique values of ultrafiltration coefficient $\left(K_{f}\right)$ was possible because filtration pressure disequilibrium was observed in all animals studied. In enalapril-treated rats (group 2) $K_{\mathrm{f}}$ averaged $0.126 \pm 0.027 \mathrm{nl} / \mathrm{s}$ per $\mathrm{mmHg}$, a value importantly higher than that observed for untreated group $1(0.061 \pm 0.023 \mathrm{nl} / \mathrm{s}$ per $\mathrm{mmHg}, P<0.01$ ).

Renal function and dextran clearance studies. Whole-animal and kidney functional parameters measured in groups 3, 4, 5, and 6 are reported in Table II. No significant differences were observed in body and kidney weight between untreated (group 3) and enalapril-treated rats (group 4), whereas $\overline{\mathrm{AP}}$ during anesthesia was significantly lower $(P<0.01)$ in treated animals. Protein concentration in arterial plasma was comparable in the two groups. Whole-kidney functional parameters in groups 3 and 4 paralleled the results of single nephron hemodynamic studies in that GFR was significantly higher in enalapril-treated rats (group 4) as compared with untreated 
Table I. (Continued)

\begin{tabular}{|c|c|c|c|c|c|c|c|c|c|c|}
\hline$C_{\hat{A}}$ & $C_{E}$ & $\pi_{A}$ & $\pi_{E}$ & SNGFR & SNFF & $Q_{A}$ & $\mathbf{R}_{\mathrm{A}} \times 10^{10}$ & $R_{E} \times 10^{10}$ & $\mathrm{R}_{\mathrm{T}} \times 10^{10}$ & $K_{\mathrm{f}}$ \\
\hline$g / d l$ & $g / d l$ & $m m H g$ & $m m H g$ & $n l / m i n$ & & $n l / m i n$ & & $d y n \cdot s \cdot \mathrm{cm}^{-s}$ & & $\mathrm{nl} / \mathrm{s}$ per $\mathrm{mmHg}$ \\
\hline $5.3 \pm 0.8$ & $7.5 \pm 1.0$ & $17 \pm 4$ & $29 \pm 6$ & $46.9 \pm 4.0$ & $0.29 \pm 0.02$ & $161 \pm 20$ & $1.94 \pm 0.11$ & $0.88 \pm 0.11$ & $2.82 \pm 0.20$ & $0.061 \pm 0.023$ \\
\hline $5.2 \pm 0.3$ & $7.6 \pm 0.4$ & $17 \pm 1$ & $29 \pm 3$ & $54.6 \pm 7.1$ & $0.31 \pm 0.03$ & $178 \pm 29$ & $1.50 \pm 0.19$ & $0.65 \pm 0.12$ & $2.14 \pm 0.25$ & $0.126 \pm 0.027$ \\
\hline NS & NS & NS & NS & $<0.05$ & NS & NS & $<0.001$ & $<0.001$ & $<0.001$ & $<0.001$ \\
\hline
\end{tabular}

rats (group 3) whereas RPF and FF only numerically increased. The same pattern was observed when GFR and RPF data were expressed for $100 \mathrm{~g}$ of animal body weight. Fractional clearance values for dextrans of graded molecular size are represented in Fig. 4. In group 4 animals the clearance of dextran molecules relative to that of inulin was significantly reduced, as compared with untreated animals (group 3), for all the molecular sizes studied (from 26 to $64 \AA$ ). It can be noted that in a logaritmic scale (see Fig. 4) enalapril treatment reduced at a similar extent glomerular filtration of relatively small, medium, and large macromolecules.

Estimates of whole-kidney function in animals of groups 5 and 6 are also reported in Table II. Untreated and enalapriltreated rats showed comparable values of body weight, kidney weight, and protein concentration in arterial plasma. Wholekidney GFR was only numerically higher in enalapril-treated rats, by the end of the study, but the difference did not reach statistical significance. Also, RPF and FF were comparable in these two groups of rats. Comparing the results of the studies performed at the 2nd mo (groups 3 and 4) with those performed at the 6th mo (groups 5 and 6), no significant difference were observed in GFR, whereas RPF in enalapril-treated animals tended to decrease as observation time increased. Mean values of FF were significantly increased in animals studied at the 6th mo than in animals studied at the 2nd mo, for treated and untreated groups.

Membrane pore parameters. Membrane pore-size distribution parameters $u, s$, and $r^{*}(1 \%)$, calculated for groups 3 and
4, are summarized in Table III. Mean pore radius of the corresponding normal pore-size distribution $u$ was significantly reduced $(P<0.005)$ by $C E$ inhibitor treatment of $\sim 5 \AA$ (from 43.2 to $38.3 \AA$ for groups 3 and 4 , respectively). On the contrary, the spreading of the distribution $s$ increased $(P<0.05)$ in enalapril-treated rats as compared with control rats. To better represent the difference in pore size between the two groups of rats their pore size distribution function $g(r)$, calculated for the mean values of $u$ and $s$ reported in Table III, are represented in Fig. 5. It can be noted that enalapril treatment uniformly reduced the radius of almost all membrane pores. Also, the parameter $r^{*}(1 \%)$, which quantifies the presence of large membrane pores, was significantly reduced in enalapril-treated rats as compared with control animals $(70.8 \pm 1.6$ vs. $72.6 \pm 1.1 \AA, P$ $<0.05)$. However, this reduction $(\approx 1.8 \AA)$ was less important than that calculated for the mean pore radius $u(\approx 4.9 \AA)$, suggesting that $C E$ inhibition reduced predominantly the dimension of medium-sized pores.

Morphological studies. After 2 mo of observation untreated rats (group 1) tended to develop focal and segmental glomerular sclerosis (five rats out of eight, see Fig. 6) and formation of tubular casts (seven rats out of eight, mean score $0.7 \pm 0.4$ ). No signs of interstitial fibrosis or inflammation were observed in these animals. Rats treated with CE inhibitor for 2 mo did not develop renal lesions, had no signs of tubulo-interstitial damage, and were almost completely free of sclerosis (only 1 rat out of 10 showed small sclerotic lesions in $1 \%$ of glomeruli). By the end of the study all untreated rats (group 5)

Table II. Body Weight, Kidney Weight, 24-h Urinary Protein Excretion, Plasma Protein Concentration, and Whole-Kidney Functional Parameters

\begin{tabular}{|c|c|c|c|c|c|c|c|c|c|c|c|}
\hline \multirow{2}{*}{ Group } & \multirow{2}{*}{$n$} & Body wt & Kidney wt & Uprot V & $\overline{\mathbf{A P}}$ & $\mathrm{C}_{\mathrm{A}}$ & \multicolumn{2}{|c|}{ GFR } & \multicolumn{2}{|c|}{ RPF } & \multirow{2}{*}{ FF } \\
\hline & & $g$ & $g$ & $m g / 24 h$ & $\mathrm{mmHg}$ & $g / d l$ & $\mathrm{ml} / \mathrm{min}$ & $\begin{array}{c}\mathrm{ml} / \mathrm{min} \mathrm{per} \\
100 \mathrm{~g}\end{array}$ & $\mathrm{ml} / \mathrm{min}$ & $\begin{array}{c}\text { ml/min per } \\
\quad 100 \mathrm{~g}\end{array}$ & \\
\hline 3. Control & 7 & $385 \pm 30$ & $1.13 \pm 0.09$ & $155 \pm 55$ & $136 \pm 10$ & $5.5 \pm 0.2$ & $1.70 \pm 0.22$ & $0.44 \pm 0.06$ & $9.4 \pm 2.7$ & $2.44 \pm 0.73$ & $0.19 \pm 0.06$ \\
\hline 4. Enalapril & 8 & $373 \pm 13$ & $1.18 \pm 0.08$ & $50 \pm 17$ & $114 \pm 14$ & $5.6 \pm 0.3$ & $2.17 \pm 0.28$ & $0.58 \pm 0.05$ & $10.6 \pm 1.6$ & $2.84 \pm 0.39$ & $0.21 \pm 0.03$ \\
\hline 5. Control & 8 & $433 \pm 26$ & $1.24 \pm 0.18$ & $295 \pm 64$ & $146 \pm 6$ & $5.6 \pm 0.5$ & $1.94 \pm 0.47$ & $0.44 \pm 0.11$ & $7.4 \pm 0.8$ & $1.72 \pm 0.19$ & $0.27 \pm 0.04$ \\
\hline 6. Enalapril & 6 & $442 \pm 18$ & $1.22 \pm 0.11$ & $128 \pm 24$ & $127 \pm 7$ & $5.6 \pm 0.4$ & $2.37 \pm 0.26$ & $0.54 \pm 0.07$ & $7.6 \pm 0.8$ & $1.71 \pm 0.23$ & $0.30 \pm 0.04$ \\
\hline$P 3$ vs. 4 & & NS & NS & $<0.01$ & $<0.01$ & NS & $<0.05$ & $<0.01$ & NS & NS & NS \\
\hline$P 5$ vs. 6 & & NS & NS & $<0.01$ & $<0.01$ & NS & NS & NS & NS & NS & NS \\
\hline$P 3$ vs. 5 & & $<0.01$ & NS & $<0.01$ & NS & NS & NS & NS & NS & $<0.05$ & $<0.05$ \\
\hline$P 4$ vs. 6 & & $<0.01$ & NS & $<0.01$ & NS & NS & NS & NS & $<0.01$ & $<0.01$ & $<0.01$ \\
\hline
\end{tabular}

Abbreviations used in this table: Uprot $\mathrm{V}$, urinary protein excretion rate; $\mathrm{C}_{\mathrm{A}}$, arterial plasma protein concentration. Values are mean $\pm \mathrm{SD}$. 


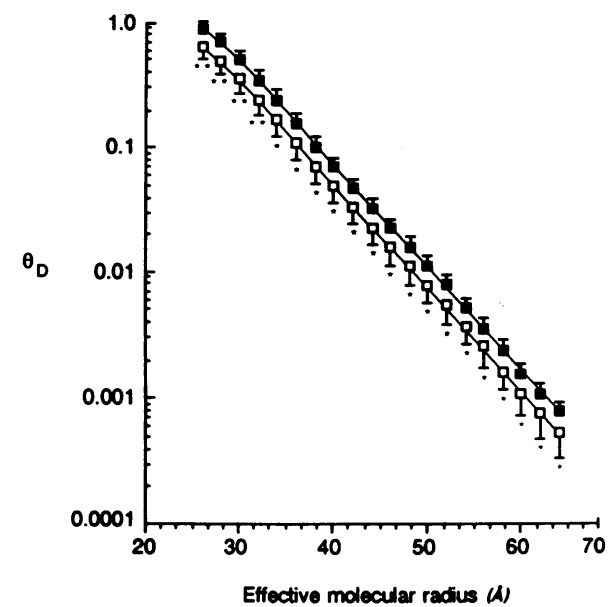

Figure 4. Fractional clearance of neutral dextran in ( $\boldsymbol{\sigma})$ control (group 3) and () enalapril-treated (group 4) rats for dextran molecules of graded molecular sizes. Values are means \pm SD. (*) $P<0.05$ vs. control group. (**) $P<0.01$ vs. control group.

developed glomerular sclerosis (see Fig. 6) affecting in average $28 \pm 15 \%$ of the glomerular population. Sclerotic changes were associated with formation of tubular casts (mean score $1.4 \pm 0.5$ ) and interstitial changes (mean score $1.0 \pm 0.5$ ). In enalapril-treated rats (group 6) at the corresponding time glomerular sclerotic lesions were significantly prevented (mean percentage of glomeruli affected by sclerosis $4.2 \pm 3.5 \%$ ) as compared to untreated group $5(P<0.01)$. Enalapril treatment also partially protected from development of tubular cast formation (average score $1.0 \pm 0.6, P<0.02$ ) and interstitial damage (mean score $0.4 \pm 0.3, P<0.02$ ).

Glomerular volume. The results of the morphometrical analysis are reported in Fig. 7. Mean GV averaged $0.84 \pm 0.12$ $\mu \mathrm{m}^{3} \times 10^{-6}$ in control rats at the 2 nd mo (group 1) and increased significantly by the end of the study (group 5: $\left.1.03 \pm 0.13 \mu \mathrm{m}^{3} \times 10^{-6}, P<0.01\right)$. No significant differences in GV were observed between enalapril and untreated rats at the 2nd mo $\left(0.82 \pm 0.13\right.$ vs. $0.84 \pm 0.12 \mu \mathrm{m}^{3} \times 10^{-6}$, respectively). Enalapril-treated rats did not show significant increase in $\mathbf{G V}$ by the end of the study $\left(0.82 \pm 0.13 \mathrm{vs.} 0.93 \pm 0.15 \mu \mathrm{m}^{3} \times 10^{-6}\right)$; thus enalapril treatment was associated with a lack of significant glomerular hyperthropy.

\section{Discussion}

Our results demonstrated that chronic administration of an angiotensin CE inhibitor, enalapril, ameliorated the progres-

Table III. Optimal Pore-Size Distribution Parameters

\begin{tabular}{lcccc}
\hline \multicolumn{1}{c}{ Group } & \multicolumn{1}{c}{$u$} & $s$ & $r^{*}(1 \%)$ \\
\hline & & $A$ & & $A$ \\
3. Control & $n=7$ & $43.2 \pm 2.1$ & $1.19 \pm 0.01$ & $72.6 \pm 11.1$ \\
4. Enalapril & $n=8$ & $38.3 \pm 3.2$ & $1.22 \pm 0.02$ & $70.8 \pm 15.7$ \\
$P$ 3 vs. 4 & & $<0.005$ & $<0.05$ & $<0.05$ \\
& & & & \\
\hline
\end{tabular}

Values are mean $\pm \mathrm{SD}$.

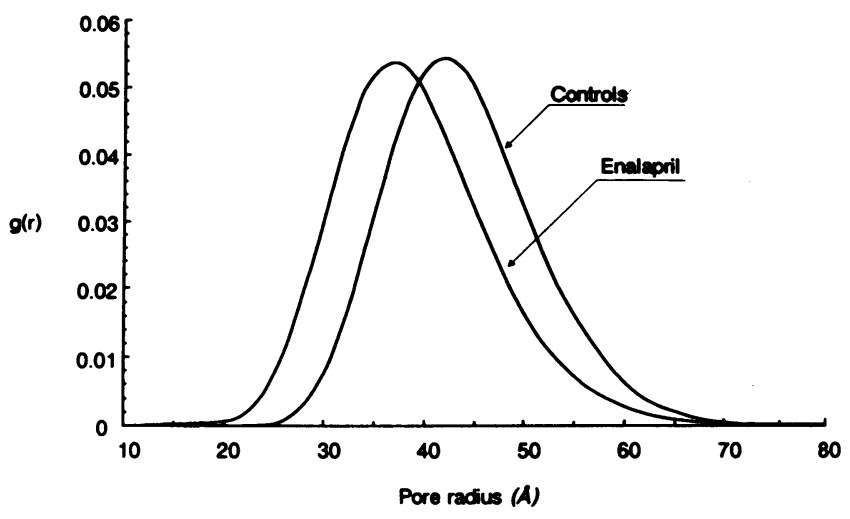

Figure 5. Pore radii distribution function $g(r)$ for calculated optimal parameter mean values of $u$ and $s$, in control (group 3 ) and in enalapril-treated (group 4) rats reported in Table III.

sion of glomerular injury in male $\mathrm{MWF} / \mathrm{Ztm}$ rats by significantly reducing proteinuria and glomerulosclerosis. The protective effect of angiotensin $\mathrm{CE}$ inhibitors on proteinuria and glomerulosclerosis has been firstly reported in rats with extensive removal of renal mass and streptozotocin-induced diabetes $(5,6)$. In both models the favorable effect of enalapril was attributed to its property of normalizing glomerular capillary hydraulic pressure, which is considered the key factor in the initiation and progression of the glomerular disease (7). This interpretation for the protective effect of $\mathrm{CE}$ inhibitors has been challenged by more recent studies. Marinides et al. (33) reported that $\mathrm{CE}$ inhibitor enalapril did not affect proteinuria and glomerulosclerosis in animals treated with repeated injections of puromycin. The same result was obtained by Scholey et al. (34) in adriamycin-treated animals and more recently confirmed by Beukers and co-workers (35), who found that $C E$ inhibition by captopril had no beneficial effect on proteinuria and development of glomerular structural damage and sclerosis in adriamycin nephrosis. In another study (8) both models were used to show that intraglomerular capillary pressure levels remained within the normal range after the disease was established and were not affected by captopril which, however, effectively prevented glomerular sclerotic lesions. These latter findings prompted the authors to suggest that at least in some progressive glomerular diseases a CE inhibitor-sensitive mechanism(s) operates independently of glomerular capillary pres-

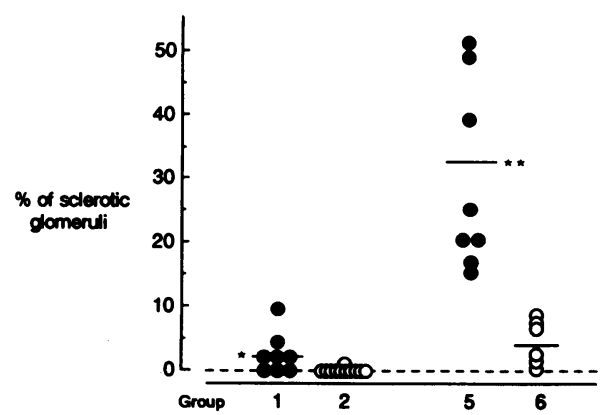

Figure 6. Percentage of sclerotic glomeruli in individual animals of (๑) control and (o) enalapril-treated groups at the 2nd mo (group 1 and 2 ) and at the 6 th mo of observation (groups 5 and 6). (*) $P$ $<0.05$ vs. group 2 . (**) $P<0.01$ vs. group 6. 


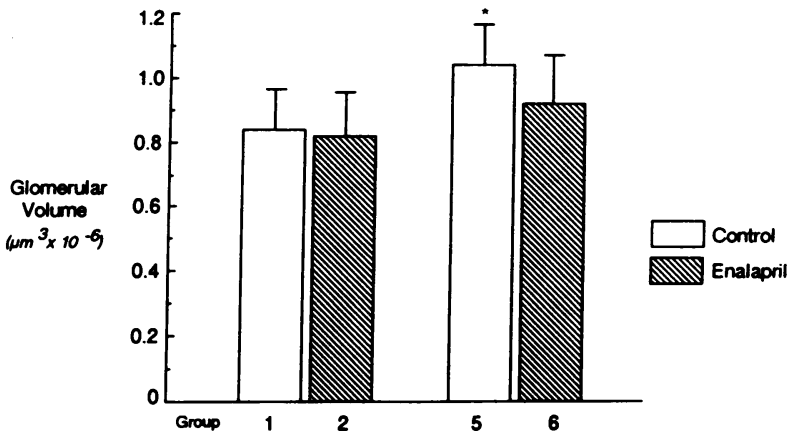

Figure 7. Glomerular volume measurements after 2 mo (groups 1 and 2) and after 6 mo (groups 5 and 6 ) of enalapril or no treatment. Bar represents means \pm SD. $(*) P<0.01$ vs. untreated group 1 at the 2nd mo.

sure. At variance, in a slightly different model of puromycin nephrosis (32), CE inhibition normalized glomerular capillary pressure and limited proteinuria and glomerulosclerosis.

In male MWF/Ztm rats, used in the present study, massive proteinuria and glomerulosclerosis were not associated with glomerular capillary hypertension. In this model enalapril treatment significantly reduced $\overline{\Delta P}$ to values lower than the normal range $(12,13)$. Whether this reduction in $\overline{\Delta \mathrm{P}}$ was the responsible factor for the protection against proteinuria and glomerulosclerosis is an open possibility, but remains matter of speculation. In fact, findings that male and female MWF/ $\mathrm{Ztm}$ rats have comparable $\overline{\Delta \mathrm{P}}$, whereas glomerular damage develops only in males (11), would indicate that glomerular damage and $\overline{\Delta \mathrm{P}}$ are not directly related in this model. However a sex-related predisposition to pressure-induced injury in this model cannot be definitely ruled out.

In addition to its effect on glomerular capillary pressure, we have found that enalapril ameliorates the size-selective function of the glomerular membrane, as indicated by a significant decrease in filtration of dextran macromolecules of graded sizes, with effective molecular radius ranging from 26 to $64 \AA$. The observation that enalapril modifies glomerular membrane permeability properties is consistent with the recent findings of Morelli et al. (9) who showed that, in insulindependent diabetic humans, enalapril exerts an antiproteinuric effect by shifting glomerular hypothetical pores towards smaller sizes, thus enhancing membrane size-selective function. The pattern of glomerular sieving curve alteration found in humans (9) and by our present study in the rat appears unique of enalapril treatment. We found that $\theta$ of small (radius < $30 \AA$ ) and large test macromolecules (radius > $50 \AA$ ) were significantly reduced while in the majority of other studies, performed in animals $(23,36,37)$ and in humans $(27,38,39)$, restoration of glomerular selective function was invariably associated with a reduction in $\theta$ of largest dextran molecules, as in the present study, but with a concomitant increase in $\theta$ of small dextran molecules.

To determine whether $\mathrm{CE}$ inhibition reduced filtration of neutral macromolecules, in this peculiar way, simply by its effects on convective and/or diffusive driving forces across the glomerular membrane or by a direct effect on membrane permeability to macromolecules, we have adopted a theoretical approach, originally developed by Deen et al. (27), that takes into account variations in solute concentrations and fluxes along the length of the glomerular capillary. This model (23) is based on the assumption that the glomerular membrane is perforated by cylindrical pores having a countinuous (lognormal) distribution of pore radii and allows characterization of intrinsic membrane pore size parameters. On the basis of the transport theory (40) one would have predict that the reduction in glomerular capillary pressure associated with enalapril treatment, as compared to untreated animals, would have result in enhanced filtration of dextran molecules, especially for small dextran molecules (radius ranging from 26 to $36 \AA$ ) that are most affected by changes in $P_{G C}$. The finding that, on the contrary, $\theta$ of small dextran molecules was decreased by $\mathrm{CE}$ inhibition indicates that enalapril directly affected intrinsic membrane permeabilities properties. Actually, theoretical analysis of experimental data documented that $\mathrm{CE}$ inhibition uniformly reduced the radius of all membrane pores, as shown by the calculated pore size distribution functions (see Fig. 4), and that this reduction was predominant for pores having radii around the mean.

These observations may be taken to suggest that enalapril directly interferes with glomerular membrane permeability properties. It has been previously observed that elevation of endogenous angiotensin II by partial renal vein constriction in the rat impaired glomerular size selectivity (25), whereas an angiotensin II antagonist, salarasin, largely neutralized such an effect. These results would suggest that enalapril may have ameliorated membrane selective properties in the present study simply by lowering intrarenal angiotensin II concentrations.

Beside reducing the urinary protein excretion rate, enalapril protected rats from the development of glomerular sclerosis. Despite the recent evidence that proteinuria does not originate from sclerotic glomeruli in rats with reduction of renal mass (41), studies on adriamycin and age-related glomerulosclerosis would suggest a possible casual relationship between proteinuria and glomerulosclerosis $(42,43)$. Actually an increased traffic of plasma macromolecules across the glomerular capillary wall is believed to induce mesangial protein overload and increase production of mesangial matrix that can alter structure and function of the glomerular basement membrane leading to development of sclerotic lesions $(7,44,45)$. It is therefore not surprising that enalapril, reducing protein filtration across the glomerular barrier, also attenuated the development of glomerular sclerotic lesions.

It has also been suggested that glomerular hypertrophy is a crucial factor leading to development of glomerular injury (46-48). In the present study after 2 mo of treatment, despite being protected completely from proteinuria by enalapril, no differences in GV were observed between treated and untreated rats. However, by the end of the study enalapril-treated animals, partiallý protected from proteinuria and sclerosis, did not develop significant glomerular hypertrophy as compared to untreated animals. These result indicate that the $\mathrm{CE}$ inhibition, beside protecting from glomerulosclerosis, do prevent glomerular hypertrophy, raising the possibility of a cause and effect relationship between the two phenomena as recently reported by Fries et al. (48).

A basic finding of the present study was that despite the decrease in glomerular capillary pressure, in the enalapril vs. control group, both SNGFR and whole-kidney GFR did not decrease with CE inhibition but rather increased, to a significant extent, thanks to a twofold increase in ultrafiltration coef- 
ficient $\left(K_{f}\right)$. These findings are in line with previous reports on the effects of enalapril in other experimental models of glomerular injury in which prevention of glomerular capillary hypertension was constantly associated with an increase in $K_{\mathrm{f}}$. Actually, in the remnant kidney model (5) enalapril-treated rats showed a lower mean value of $\overline{\Delta \mathrm{P}}$, as compared with untreated rats (36 vs. $50 \mathrm{mmHg}$ ), whereas $\bar{K}_{\mathrm{f}}$ was significantly higher ( 0.096 vs. $0.049 \mathrm{nl} / \mathrm{s}$ per $\mathrm{mmHg}$ ). The same pattern of data was obtained in the experimental model of diabetes (6), $\overline{\Delta \mathrm{P}}$ was lower in enalapril-treated rats $\left(37 \mathrm{vs} .52 \mathrm{mmHg}\right.$ ) and $K_{\mathrm{f}}$ higher (0.101 vs. $0.057 \mathrm{nl} / \mathrm{s}$ per $\mathrm{mmHg})$ as compared to untreated animals. Finally, also in the study of Anderson et al. (32), enalapril treatment in the late phase of puromycin aminonucleoside nephrosis prevented glomerular hypertension (mean $\overline{\Delta \mathrm{P}}$ values: 31 vs. $48 \mathrm{mmHg}$ ) and allowed the maintenance of $K_{\mathrm{f}}(\leq 0.108 \mathrm{vs} .0 .039 \mathrm{nl} / \mathrm{s}$ per $\mathrm{mmHg}$, respectively, enalapril vs. untreated group). The constant finding of elevated $K_{\mathrm{f}}$ in enalapril vs. the respective control animals could be due to an increase in hydraulic permeability of the glomerular membrane or to an increase in capillary surface area available for filtration, the two determinants of the $K_{\mathrm{f}}$. With the present study we established in the same animals that underwent micropuncture studies that enalapril did not affect GV. Since an important increase in glomerular filtering surface area cannot occur without a parallel increase in GV these findings would suggest that the observed twofold increase in $K_{\mathrm{f}}$ associated with $\mathrm{CE}$ inhibition must result from predominant elevation of hydraulic membrane permeability.

Altogether, the present results indicate that $\mathrm{CE}$ inhibition not only affects glomerular microcirculation but also directly influences glomerular membrane functional properties by reducing membrane pore sizes and concomitantly increasing hydraulic permeability of the glomerular capillary wall. This rises the question whether the beneficial effect of enalapril on renal disease progression, which in the past has been attributed to its properties of preventing glomerular hypertension, is not rather related to a concomitant preservation of glomerular membrane size-selective and hydraulic permeability functions. This possibility opens new insights into the mechanisms responsible for the protective effect of CE inhibitors on the progression of experimental and human nephropathies.

In summary, our study indicates that in a model of spontaneous progressive glomerulosclerosis: $(a)$ enalapril protected against development of proteinuria and glomerular injury; $(b)$ the protective effects of enalapril was associated with a reduction in glomerular capillary pressure to values significantly lower than the normal range; (c) CE inhibition enhanced sizeselective function reducing mean radius of the membrane pore population and significantly enhanced glomerular hydraulic permeability properties.

\section{Acknowledgments}

We thank Dr. B. Brugnetti for excellent assistance during micropuncture studies. L. Piccoli helped prepare the manuscript. Enalapril was a gift from Dr. L. Carratelli, Merck, Sharp \& Dohme Italia SpA.

This work was partially supported by a grant from the Italian National Research Council (No. 87.00155.04).

\section{References}

1. Hostetter, T. H., J. L. Olson, H. G. Rennke, M. A. Venkatachalam, and B. M. Brenner. 1974. Hyperfiltration in remnant nephrons: a potentially adverse response to renal ablation. Am. J. Physiol. 241:F85-F93.
2. Anderson, S., T. W. Meyer, H. G. Rennke, and B. M. Brennner. 1985. Control of glomerular hypertension limits glomerular injury in rats with reduced renal mass. J. Clin. Invest. 76:612-619.

3. Hostetter, T. H., J. L. Troy, and B. M. Brennner. 1981. Glomerular hemodynamics in experimental diabetes mellitus. Kidney Int. 19:410-415.

4. Zatz, R., T. W. Meyer, H. G. Rennke, and B. M. Brennner. 1985. Predominance of hemodynamic rather than metabolic factors in the pathogenesis of diabetic glomerulopathy. Proc. Natl. Acad. Sci. USA. 82:5963-5967.

5. Anderson, S., H. G. Renneke, and B. M. Brenner. 1986. Therapeutic advantage of converting enzyme inhibitors in arresting progressive renal disease associated with systemic hypertension in the rat. $J$. Clin. Invest. 77:1993-2000.

6. Zatz, R., B. R. Dunn, T. W. Meyer, S. Anderson, H. R. Rennke, and B. M. Brenner. 1986. Prevention of diabetic glomerulopathy by pharmacological amelioration of glomerular capillary hypertension. $J$. Clin. Invest. 77:1925-1930.

7. Brenner, B. M., T. W. Meyer, and T. H. Hostetter. 1982. Dietary protein intake and progressive nature of kidney disease: the role of hemodynamically mediated injury in the poathogenesis of progressive glomerular sclerosis in aging, renal ablation and intrinsic renal disease. N. Engl. J. Med. 307:652-659.

8. Fogo, A., Y. Yoshida, A. D. Glick, T. Homma, and I. Ichikawa 1988. Serial micropuncture analysis of glomerular function in two rat models of glomerular sclerosis. J. Clin. Invest. 82:322-330.

9. Morelli, E., N. R. Loon, and B. D. Myers. 1989. Effect of converting enzyme inhibitor (CEI) on diabetic glomerular injury (DGI). Kidney Int. 35:211. (Abstr.)

10. Hackbarth, H., D. Buttern, D. Jarck, M. Pothmann, C. Messow, and K. Gartner. 1983. Distribution of glomeruli in the renal cortex of Munich Wistar Fromter (MWF) rats. Renal Physiol. 6:63-71.

11. Remuzzi, A., S. Puntorieri, A. Mazzoleni, and G. Remuzzi. 1988. Sex-related differences in glomerular ultrafiltration and proteinuria in Munich-Wistar rats. Kidney Int. 34:481-486.

12. Ichikawa, I., D. A. Maddox, M. G. Cogan, and B. M. Brenner. 1978. Dynamics of glomerular ultrafiltration in euvolemic MunichWistar rats. Renal Physiol. 1:121-131.

13. Arendshorst, W. J., and C. W. Gottschalk. 1985. Glomerular ultrafiltration dynamics: historical perspective. Am. J. Physiol. 248:F163-F174.

14. Pfeffer, J. M., M. A. Pfeffer, and E. D. Frohlich. 1971. Validity of an indirect tailcuff method for determining systolic arterial pressure in unanesthetized normotensive and spontaneously hypertensive rats. J. Lab. Clin. Med. 78:957-962.

15. Read, S. M., and D. H. Northcote. 1981. Minimization of variation in the response to different of the Coomassie blue $\mathbf{G}$ dyebinding assay for protein. Anal. Biochem. 116:53-64.

16. Vurek, G. C., and S. E. Pegram. 1966. Fluorimetric method for the determination of nanogram quantities of Inulin. Anal. Biochem. 16:409-419.

17. Benson, J. R., and P. E. Hare. 1975. O-phthalaldehyde: fluorogenic detection of prymary ammines in the picomole range: Comparison with fluores-camine and ninhydryn. Proc. Natl. Acad. Sci. USA. 72:619-622.

18. Viets, J. W., W. M. Deen, J. L. Troy, B. M. Brenner. 1978. Determination of serum protein concentration in nanoliter blood samples using fluorescamine or o-phthalaldehyde. Anal. Biochem. 88:513-521.

19. Fuehr, J., J. Kaczmarczyk, and C. D. Kruttgen. 1955. Eine einfache colorimetrische Methode zur Inulinbestimmung für NierenClearance-Untersuchungen bei Stoffwechselgesunden und Diabetikern. Klin. Wochenschr. 33:729-730.

20. Lowry, O. H., N. J. Rosebrough, A. L. Farr, and R. J. Randall. 1951. Protein measurement with the Folin phenol reagent. J. Biol. Chem. 193:265-275.

21. Deen, W. M., J. L. Troy, C. R. Robertson, and B. M. Brenner. 
1973. Dynamics of glomerular ultrafiltration in the rat. IV. Determination of the ultrafiltration coefficient. J. Clin. Invest. 52:1500-1508.

22. Deen, W. M., C. R. Robertson, and B. M. Brenner. 1972. A model of glomerular ultrafiltration in the rat. Am. J. Physiol. 233:F1178-F1183.

23. Remuzzi, A., C. Battaglia, L. Rossi, C. Zoja, and G. Remuzzi. 1987. Glomerular size selectivity in nephrotic rats exposed to diets with protein content. Am. J. Physiol. 253:F318-F327.

24. Smith, H. W., N. Finkelstein, and L. Aliminosa. 1945. The renal clearances of substitute hippuric acid derivates and other aromatic acids in dogs and man. J. Clin. Invest. 24:388-404.

25. Yoshioka, T., T. Mitarai, V. Kon, W. M. Deen, H. G. Rennke, and I. Ichikawa. 1986. Role for angiotensin II in an overt functional proteinuria. Kidney Int. 30:538-545.

26. Granath, K. A., and B. E. Kvist. 1967. Molecular weight distribution analysis by gel chromatography on sephadex. J. Chromatogr. 28:69-81.

27. Deen, W. M., C. R. Bridges, B. M. Brenner, and B. D. Myers. 1985. Heteroporous model of glomerular size selectivity: application to normal and nephrotic humans. Am. J. Physiol. 249:F374-F389.

28. Sacchi Landriani, G., V. Guardabasso, and M. Rocchetti. 1983. NL-FIT: a microcomputer program for nonlinear fitting. Comput. Programs Biomed. 16:35-42.

29. Bertani, T., A. Poggi, R. Pozzoni, F. Delaini, G. Sacchi, Y. Thoua, G. Mecca, G. Remuzzi, and M. B. Donati. 1982. Adriamycininduced nephrotic syndrome in rats. Sequence of pathological events. Lab. Invest. 46:16-23.

30. Weibel, E. R. 1979. Stereological Methods: Practical Methods for Biological Morphometry. Volume 1. Academic Press, Ltd., London. $1-145$.

31. Wallenstein, S., C. L. Zucker, and J. L. Fleiss. 1980. Some statistical methods udseful in circulation research. Circ. Res. 47:1-9.

32. Anderson, S., J. R. Diamond, M. J. Karnovsky, and B. M. Brenner. 1988. Mechanisms underlying transition from acute glomerular injury to late glomerular sclerosis in a rat model of nephrotic syndrome. J. Clin. Invest. 82:1757-1768.

33. Marinides, G. N., G. C. Groggel, A. H. Cohen, T. Cook, R. L. Baranowski, C. Westenfelder, and W. A. Border. 1987. Failure of angiotensin converting enzyme inhibition to affect the course of chronic puromycin aminonucleoside nephropathy. Am. J. Pathol. 129:394-401.

34. Scholey, J. W., and T. W. Meyer. 1987. Reducing glomerular capillary pressure does not reduce proteinuria in rats with adriamycin nephrosis. Kidney Int. 31:393. (Abstr.)

35. Beukers, J. J. B., P. J. Hoedemaeker, and J. J. Weening. 1988. A comparison of the effects of converting-enzyme inhibition and protein restriction in experimental nephrosis. Lab. Invest. 59:631-640.

36. Bhorer, M. P., C. Baylis, C. R. Robertson, and B. M. Brenner. 1977. Mechanisms of the Puromycin-induced defects in the transglomerular passage of water and macromolecules. J. Clin. Invest. 60:152-161.

37. Weening, J. J., and H. G. Rennke. 1983. Glomerular permeability and polyanion in adriamycin nephrosis in the rat. Kidney Int. 24:152-159.

38. Winetz, J. A., H. V. Golbetz, R. J. Spencer, J. A. Lee, and B. D. Myers. 1982. Glomerular function in advanced diabetic nephropathy. Kidney Int. 21:750-756.

39. Shemesh, O., J. C. Ross, M. W. Deen, G. W. Grant, and B. D. Myers. 1986. Nature of the glomerular capillary injury in human membranous glomerulopathy. J. Clin. Invest. 77:868-877.

40. Deen, W. M., M. P. Bohrer, and B. M. Brenner. 1979. Macromolecule transport across glomerular capillaries: application of pore theory. Kidney Int. 16:353-365.

41. Yoshioka, T., H. Shiraga, Y. Yoshida, A. Fogo, A. D. Glick, W. M. Deen, J. R. Hoyer, and I. Ichikawa. 1988. "Intact nephrons" as the primary origin of proteinuria in chronic renal disease: study in the rat model of subtotal nephrectomy. J. Clin. Invest. 82:1614-1623.

42. Bertani, T., F. Cutillo, C. Zoja, M. Broggini, and G. Remuzzi. 1986. Tubulo-interstitial lesions mediate renal damage in adriamycin glomerulopathy. Kidney Int. 30:488-496.

43. Bertani, T., C. Zoja, M. Abbate, M. Rossini, and G. Remuzzi. 1989. Age-related nephropathy and proteinuria in rats with intact kidneies exposed to diets with different protein content. Lab. Invest. 60:197-204.

44. Grond, J., S. Marten, J. Koudstall, and J. D. Elema. 1982. Mesangial function and glomerular sclerosis in rats after unilateral nephrectomy. Kidney Int. 22:338-343.

45. Couser, W. G., and M. M. Stilmant. 1975. Mesangial lesions and focal glomerular sclerosis in the aging rat. Lab. Invest. 33:554561.

46. Yoshida, Y., A. Fogo, and I. Ichikawa. 1989. Glomerular hemodynamic changes vs. hypertrophy in experimental glomerular sclerosis. Kidney Int. 35:654-660.

47. Fogo, A., Y. Yoshida, I. Ichikawa, and A. D. Glick. 1988. Hypertrophic glomeruli and possible relationship to glomerular sclerosis. Lab. Invest. 58:31a. (Abstr.)

48. Fries, J. U., D. Sandstrom, T. W. Meyer, and H. G. Rennke. 1989. Glomerular hypertrophy and epithelial cell injury are determinants of progressive glomerulo-sclerosis in the rat. Lab. Invest. $60: 205-218$. 